\title{
Design of an ultrasonic physiotherapy system with pulse wave feedback control
}

\author{
Ran Peng ${ }^{\mathrm{a}, \mathrm{b}}$, Yang Luo ${ }^{\mathrm{a}}$, Zhangyong $\mathrm{Li}^{\mathrm{a}, *}$, Wei Wang ${ }^{\mathrm{a}}$ and Yu Pang ${ }^{\mathrm{a}}$ \\ ${ }^{a}$ Chongqing University of Posts and Telecommunications, Chongqing, China \\ ${ }^{\mathrm{b}}$ National Engineering Research Center of Ultrasound Medicine, Chongqing Medical University, \\ Chongqing, China
}

\begin{abstract}
.
BACKGROUND: Due to different physical and biological mechanisms behind ultrasound hyperthermia and phonophoresis, the requirement for ultrasound power, frequency and control modes varies.

OBJECTIVE: This paper introduces an adaptive ultrasonic physiotherapy system based on real-time surveillance over physiological characteristics of the patients, which in turn assists the individual treatment and dose limitation in auxiliary rehabilitation.

METHODS: The method essentially takes advantage of distinctive characteristics of two different phases (systole and diastole) of the human cardiac cycle as a medium for modulation. The abundance of blood flow during systole enables energy exchange for hyperthermia while blood flow insufficiency caused by diastole assists in drug penetration. Said method could improve the adjuvant therapy as it provides partial drug penetration and therapeutic dosage control.

RESULTS: By adjusting time window and intensity of multi-frequency ultrasound, it is possible to reduce the irradiation dosage to around $22 \%$ of that during continuous irradiation at $1 \mathrm{MHz}$. The method shows high potential in clinical practice.

CONCLUSION: Frequency-tuning ultrasound therapy would be more efficient regarding drug penetration and improve the therapeutic efficacy of hyperthermia.
\end{abstract}

Keywords: Ultrasonic physiotherapy, ultrasound hyperthermia, drug penetration, physiological parameter feedback, real-time control

\section{Introduction}

The mechanical, thermal, cavitation and dispersion effects of ultrasound can induce biological effects in human tissue, among which a few effects may be utilized for therapy and rehabilitation [1,2]. Recent research and clinical examinations have established safe doses for therapeutic ultrasound [3], resulting in rampant growth of its clinical applications-notably its widely recognized application in adjuvant therapy.

Previous studies on ultrasound technique in rehabilitation treatment applications were mainly focused in two directions: 1. The ultrasonic thermal effect which benefits soft tissue recovery and provides pain relief; 2. Ultrasound mechanical effects which improve drug penetration for adjuvant therapies [4]. The treatment of diseases such as postherpetic neuralgia, tuberculous pleurisy, chronic prostatitis and joint injuries would benefit significantly from the combination of both ultrasound diathermy and ultrasoundmediated drug delivery [5].

\footnotetext{
${ }^{*}$ Corresponding author: Zhangyong Li, College of Bioinformation, Chongqing University of Posts and Telecommunications, Chongwen Road 2, Nanan district, Chongqing 400065, China. Tel.: +86 2362471782; E-mail: lizy@ cqupt.edu.cn.
}

0928-7329/17/\$35.00 (c) 2017 - IOS Press and the authors. All rights reserved

This article is published online with Open Access and distributed under the terms of the Creative Commons Attribution NonCommercial License (CC-BY-NC 4.0). 


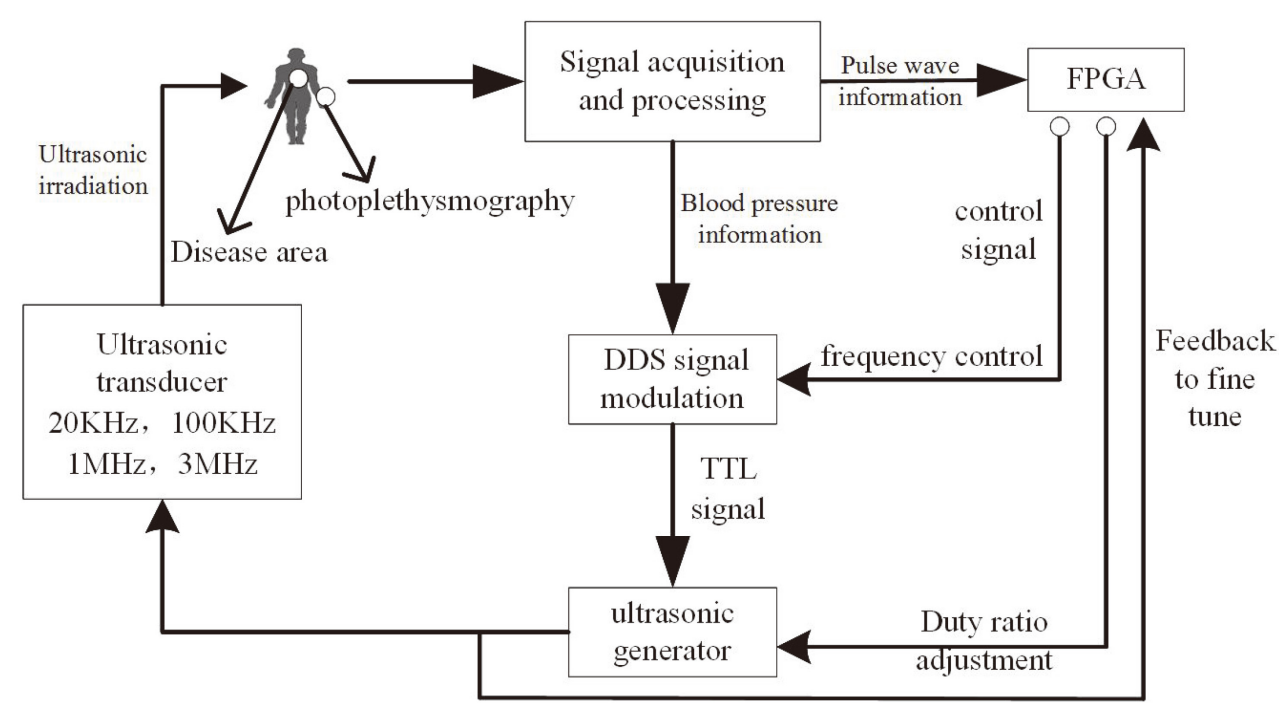

Fig. 1. System principle block diagram.

Phonophoresis relies on mechanical energy to alter the cell membrane permeability and in the process enhances cell membrane diffusion, thus making it easier for the drug to pass through the skin or mucous membrane into the body and improve the therapeutic effect [6]. Previous studies have shown that high transmission power was not necessary for medicine penetration. Selection of appropriate ultrasound frequency depends on the part of the body in need of treatment as well as the medicine adopted for the treatment. Suitable frequencies usually fall within the range of $20 \mathrm{kHz}-3 \mathrm{MHz}$ [7].

In contrast, ultrasound hyperthermia, mostly using $1 \mathrm{MHz}$ frequency and relatively high power, relies upon the conversion of ultrasonic energy into heat. In healthy tissue, $79 \% \sim 82 \%$ of the heat generated by ultrasound would disperse through blood circulation. Due to inadequate blood supply, tumors and inflamed tissue absorb a great deal of energy, which leads to cell structure damage and inhibited growth. Ultrasound hyperthermia accelerates cell metabolism of healthy tissues, thereby assisting the functional repair of normal tissues [8].

As indicated above, the characteristics of the blood supply cycle were integrated into the output control parameters. And to improve individual treatment effect and dosage limitation, we take advantage of the periods of abundant blood flow for energy conversion of hyperthermia and that of insufficient blood flow to assist drug penetration.

\section{Method and design}

The establishment of this system is to synchronize ultrasonic output with human pulse rate and to remain consistent with the body's circadian rhythm. Precise and reliable control of the output power and treatment modes of ultrasonic therapy can be achieved with the components shown in the system principle block diagram (Fig. 1).

\subsection{Overall design}

Since the control of ultrasonic intensity is mainly determined by the arterial blood volume, the finger photoplethysmography is utilized for signal acquisition of this design to detect the variations of blood 
volume [9]. The collected pulse statistics are transmitted to the ultrasonic frequency DDS (Direct Digital Synthesizer) as critical parameters of ultrasonic power control.

The field-programmable gate array (FPGA) receives the pulse waveform and performs frequency domain filtering. The information provided is an important reference for choosing the appropriate ultrasonic treatment mode, specifically for selecting the output frequency and adjusting the pulse sequence and duty ratio. These parameters are synthesized in an acoustic power control module that signals one or more of the ultrasonic transducers to work in cooperation. The system thereby selects the optimal treatment mode and dosage adjustment based on the physical condition of each patient, which enhances the treatment effect.

\subsection{Ultrasound hyperthermia and drug penetration}

Ultrasonic hyperthermia relies on the conversion of ultrasonic mechanical energy into thermal energy in biological tissues. Higher temperature causes damage to lesion tissue, while a lower amount of energy absorption promotes the rehabilitation of lesion tissue [10]. The low-intensity pulsed ultrasound (LIPUS) used in this rehabilitation technology transfers energy at an intensity less than $3 \mathrm{~W} / \mathrm{cm}^{2}$, which is not sufficient to cause a thermal effect, so it avoids the adverse reactions associated with traditional ultrasound treatment. This technique has achieved satisfactory results in rehabilitation from surgical incision, tendon injury, nerve injury and fracture, as well as regeneration or repair after radioactive bone necrosis [11].

Ultrasonic drug penetration, also called phonophoresis, utilizes the effect of ultrasonic waves on the dispersion of a medium that increases the permeability of cell membranes, helping the drug to pass through the skin or mucosal membrane into the body. This effect has been used with external therapeutic drugs in traditional Chinese medicine [12]. The application of drug directly to the treatment site helps avoid the first-pass effect and maintains its original efficacy [13].

Assuming that the stratum corneum lipid region is parallel to the surface of the skin, the ultrasonic permeability coefficient $P^{U S}$ of a small molecule $(\mathrm{Mr}<500)$ can be expressed as follows:

$$
P^{U S}=\frac{K \cdot D_{W} \cdot \Phi}{N \cdot I} .
$$

$K$ is the permeability coefficient of the drug in the water channel. $N$ is the number of molecules in the lipid region $(N=15) . I$ is the thickness of each layer of the lipid zone (usually, $I=50 \mathrm{~nm}) . \Phi$ is the area of the water channel for the lipid region, and $D_{W}$ is the drug diffusion coefficient (usually, $D_{W}=$ $5 \times 10^{-5}$ ). In the equation, $\Phi, N, I$ and $D_{W}$ are not related to the properties of the drug; therefore, the effectiveness of ultrasonic penetration is independent of the drug properties [14].

The low-frequency infiltration enhancement rate $E$ is expressed by the following formulas:

$$
\begin{aligned}
E_{\text {total }} & =E_{\text {conv }} \cdot E_{\text {diff }}, \\
E_{\text {conv }} & =\frac{P_{e}}{1-\exp \left(-P_{e}\right)}, \\
E_{\text {diff }} & =\frac{H\left(\frac{r_{p}}{r^{u s}}\right) \cdot\left(\frac{\varepsilon}{\tau}\right)^{\text {us }}}{H\left(\frac{r_{p}}{r^{\text {passive }}}\right) \cdot\left(\frac{\varepsilon}{\tau}\right)^{\text {passive }}},
\end{aligned}
$$

Where $E_{c o n v}$ is the infiltration enhancement rate caused by convection. $E_{\text {diff }}$ is the infiltration enhancement rate caused by diffusion. $P_{e}$ is the Peclet number. $\varepsilon$ represents the porosity of the stratum corneum. $\tau$ represents the distortion of the stratum corneum. $r_{p}$ is the hydration radius of the drug. $r^{u s}$ and $r^{\text {passive }}$ 


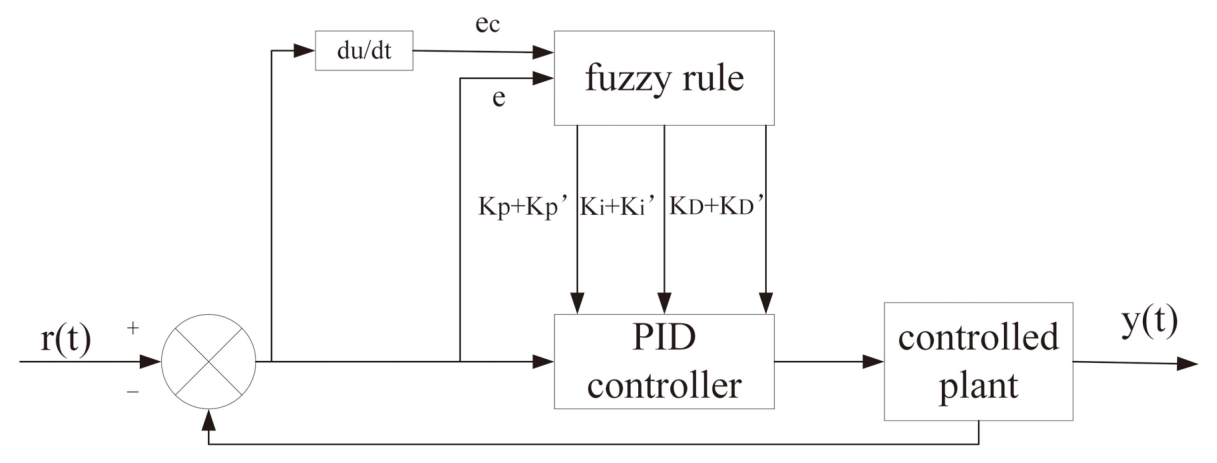

Fig. 2. Power adaptive control based on PID.

represent the effective radius of the stratum corneum water channel under ultrasonic conditions and natural condition. $H$ is the diffusion barrier factor function.

The calculations show that under low-frequency ultrasound, the drug can pass through the water channel of the disordered lipid region formed by the ultrasound. Because the structure of a keratinocyte cell is more open than that of a lipid region, the penetration is much faster in a keratinocyte cell. Therefore, the penetration frequency selection should be determined by the composition of the disordered lipid regions.

\subsection{Feedback control of PID algorithm}

In this system, the average pulse rate of the patient is used to determine the power feedback, and the ultrasonic output $P_{i}$ is calculated according to the power conversion rate of the transducer:

$$
P_{i}=K \frac{B P \times 40 \times Q \times N}{300 \times 60} .
$$

The power generator is modulated by: $P_{0}=\frac{P_{i}}{\alpha}$.

In Eq. (5), $Q$ is blood flow per heartbeat; $N$ is pulse beats per minute and $K$ is the adjusted coefficient of power. On account of the differences in individual physical fitness and the ability to withstand ultrasound intensity at the disease site, the coefficient $K$ can be adjusted in addition to the pulse feedback control.

The system determines the proportional coefficient, integration time and differential time of the proportional-integral-derivative (PID) controller according to the characteristics of the controlled process. Moreover, a fuzzy PID is applied to the automatic control of amplitude and frequency switching. The transfer function of the regulator is expressed as follow:

$$
G(S)=\frac{U(S)}{E(S)}=K_{p}+\frac{K_{i}}{S}+K_{d} S=K_{P}\left(1+\frac{1}{T_{i} S}+T_{d} S\right) .
$$

In Eq. (6), $K_{P}$ is the proportional coefficient, $K_{i}$ is the integration coefficient, $K_{d}$ is the differential coefficient, $T_{i}$ is the integral time constant and $T_{D}$ is the differential time constant.

PID control parameters are determined by calculating the pressure deviation $e$ and deviation change rate $e_{c}$ real-time and the fuzzy set is then established together with control parameters $K_{p}, K_{i}$ and $K_{d}$ (as shown in Fig. 2) [15]. In this process, the triangle and normal membership functions are applied to predict the result precisely, and the control parameters are corrected by the combination of the morphological characteristics of the pulse wave. 


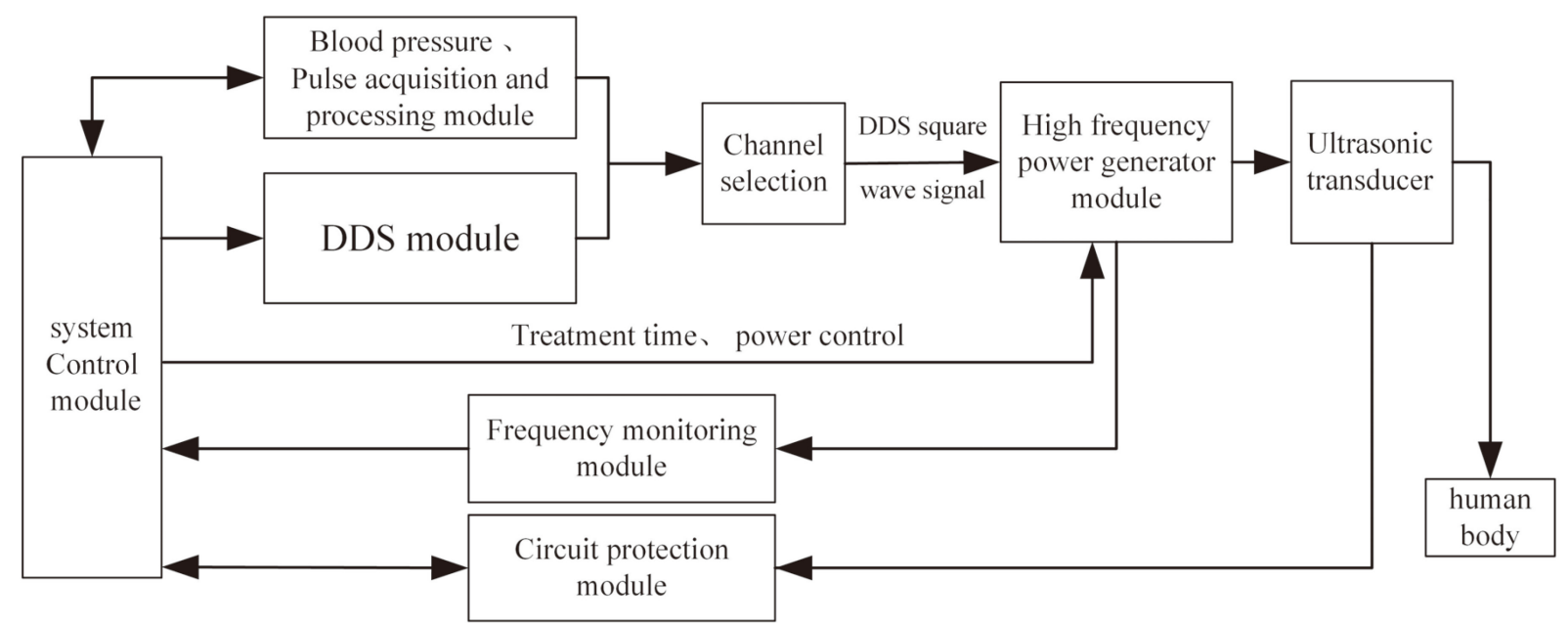

Fig. 3. System schematic block diagram.

\section{System composition}

The main hardware components of this system include a system control module, a pulse acquisition and processing module, a DDS module, a channel selection module, a high-frequency power generator, an ultrasonic transducer and a frequency monitoring module, as shown in Fig. 3.

The system control module, which employs an Altera Stratix II series FPGA (EP2S60F484) as the primary processor, coordinates the operation of the other modules and generates the ultrasonic control signal. During the signal acquisition and processing, it produces synchronous control signals containing blood volume information obtained through the acquisition of the pulse wave and delivers them to the DDS, which generates square signal. It is used to control the ultrasonic frequency, duty cycle, and repetitive cycle.

This signal switches and controls the transducer via multiplexers (Max4051), and then it is converted to a high-frequency analog signal by the high-frequency power generator. Ultimately, the transducer converts electrical energy into ultrasound effects at the disease site. Due to the difference in transmission between impedance matching and ultrasound channel, the actual frequency and amplitude should be adjusted by the feedback signal from the monitoring module to ensure precise control. The circuit protection module comprises a temperature detection circuit and an alarm circuit, which detect the temperature of the transducer anrated ensure it can work stably.

\subsection{DDS frequency source}

The DDS frequency source consists of a phase accumulator, a sine look-up table, a D/A (digital to analog) converter and a low-pass filter [16].

In Fig. $4, f_{r}$ is the reference clock frequency and $f_{0}$ is the output signal frequency. The phase accumulator receives the L-bit frequency control word which is called $P_{F S W}$, accumulating $P_{F S W}$ in each clock cycle, and then it transmits the high $\mathrm{K}$ bit of the accumulative results to the sine look-up table. The sine wave previously stored in the look-up table is then sent to the D/A converter after the system seeking, and converting phase increment to output frequency successfully. 


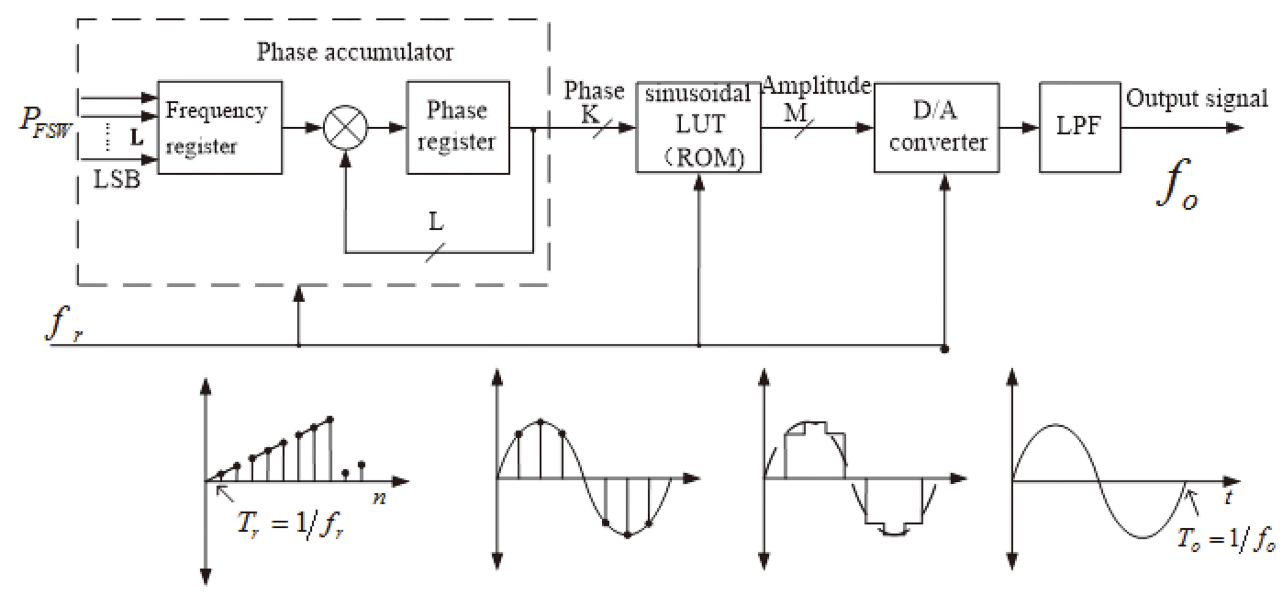

Fig. 4. DDS Work flow chart.

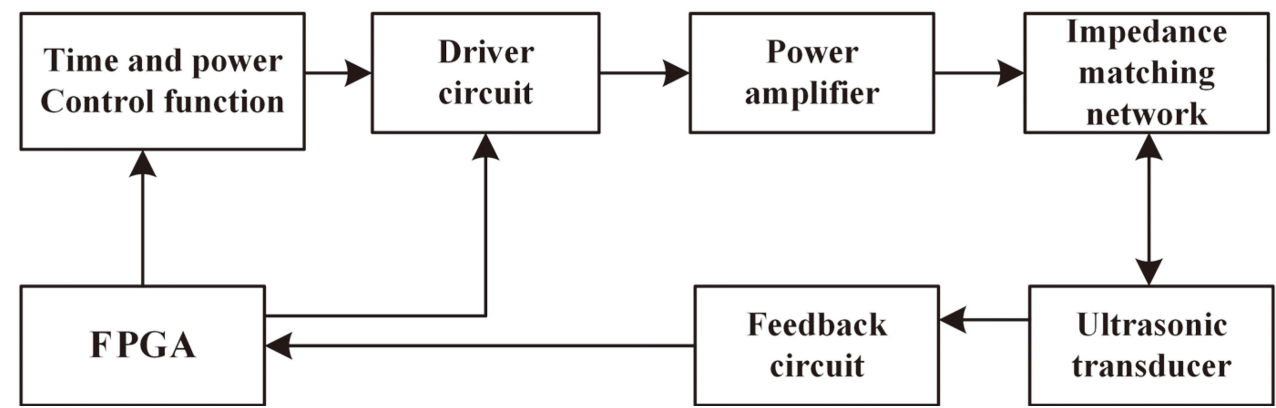

Fig. 5. Frequency feedback control block diagram.

As the phase accumulator accumulates fully, it will generate an overflow which presents a full cycle of the rotation around the center and then implements ensuing output. The cycle of the output signal is

$$
T_{o}=\frac{2^{L} T_{r}}{P_{F S W}}=\frac{1}{f_{o}} .
$$

\subsection{Power generation and monitoring}

The energy output of the ultrasonic transducer is controlled by the high-frequency power generator module, which acquires the control signal in real time through feedback calculation of the pulse wave provided by the FPGA. Different therapies combination can be achieved by altering the treatment time, power output and pulse sequence.

The system drives the power amplifier to work through the drive circuit and generates the corresponding output power, while a transmission line transformer is employed for the broadband impedance matching of the ultrasonic transducer. The feedback loop, namely the frequency monitoring module, obtains and transmits the voltage sampling and phase detection results to the FPGA for phase-sensitive detection, as shown in Fig. 5.

Phase-sensitive detection extracts weak signals of amplitude and phase from background noise, providing high flexibility, great linearity, and a strong inhibitory effect on random noise [17]. 
Table 1

System operating parameters

\begin{tabular}{lccl}
\hline Center frequency $(\mathrm{Hz})$ & Static capacitance $(\mathrm{pF})$ & Syntonic impedance $(\Omega)$ & Maximum acoustic power $\left(\mathrm{W} / \mathrm{cm}^{2}\right)$ \\
\hline $20 \mathrm{kHz}$ (Type B) & 1623 & 21.6 & 8.4 (@50\% Duty ratio) \\
$50 \mathrm{kHz}$ (Type B) & 1656 & 23.5 & 8.7 (@50\% Duty ratio) \\
$1 \mathrm{MHz}$ (Type B) & 1495 & 28.3 & 7.8 (@50\% Duty ratio) \\
$3 \mathrm{MHz}$ (Type B) & 4538 & 31.7 & 7.4 (@ 50\% Duty ratio) \\
$1 \mathrm{MHz}$ (Type A) & 1495 & 21.2 & 2.5 (Continuous output) \\
\hline
\end{tabular}

The input signal can be expressed as a superposition of $N$ components:

$$
V_{i}(n)=A \sin (2 \pi n / N+\varphi)+V_{n}(n),
$$

where $n=1,2, \ldots, N$; $\mathrm{A}$ is amplitude and $\varphi$ is phase.

According to the matched filter (MF) principle, we find:

$$
\hat{A}=\frac{2}{N} \sqrt{V_{r}^{2}+V_{q}^{2}} \quad \hat{\varphi}=\arctan \left(\frac{V_{q}}{V_{r}}\right) .
$$

In Eq. (9), $V_{r}$ is the syntropic component,

$$
V_{r}=\sum_{n=1}^{N} V_{i}(n) \sin \left(\frac{2 \pi n}{N}\right) ;
$$

and $V_{q}$ is the quadrature component,

$$
V_{q}=\sum_{n=1}^{N} V_{i}(n) \cos \left(\frac{2 \pi n}{N}\right) .
$$

The framework of digital phase-sensitive detection is based on multiplication and accumulation, which is easily realized in FPGA, in order to improve system integration and reduce power consumption. With this design, it is possible to adjust the duty cycle of the signal and precisely control the pulse sequence.

\section{Evaluation and results}

\subsection{System parameters}

Exceeding the tolerance threshold of the biological cell tissue to the dose of ultrasound therapy will cause irreversible damage to the organism [18]. In general, acoustic intensity within the range of $0.1-$ $2.5 \mathrm{~W} / \mathrm{cm}^{2}$ will meet the needs of most treatments, while continuous intensity above $3.0 \mathrm{~W} / \mathrm{cm}^{2}$ may cause damage. Low acoustic intensity has been used for treatment of sensitive biological organs, while a high instantaneous pulse (up to $8.0 \mathrm{~W} / \mathrm{cm}^{2}$ ) is occasionally applied for auxiliary drug delivery. Therefore, selection of the functional characteristics of ultrasonic transducers in the system is crucial.

The ultrasonic transducer used in this system has a diameter of $2 \mathrm{~cm}$ for hyperthermia (labeled as 'Type A'), and a diameter of $1 \mathrm{~cm}$ for phonophoresis (labeled as 'Type B'). To obtain the transducer parameters, a 4294A Agilent precision impedance analyzer (with a frequency range of $40 \mathrm{~Hz}-110 \mathrm{MHz}$ ) measured the resonant impedance and capacitance, and an HFO-660 fiber optic hydrophone system from ONDA measured the ultrasonic dose in water. The measurement results are shown in Table 1. 


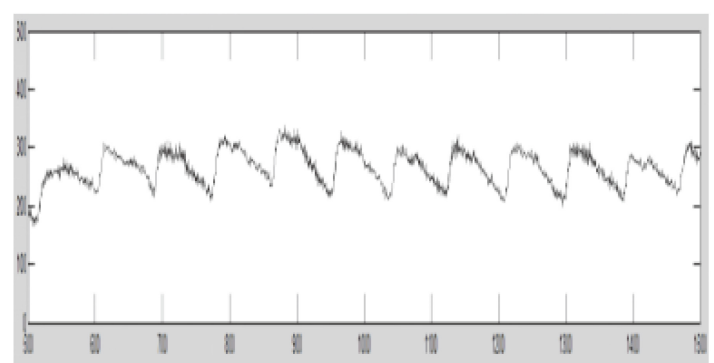

(a)

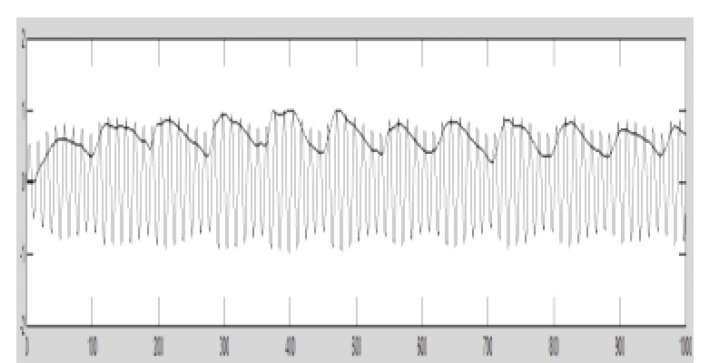

(b)

Fig. 6. (a) Original pulse waveform. (b) Control signal from modulation.

\subsection{Pulse wave acquisition and modulation}

The normal range for pulse rate is $60-100$ beats per minute. The amplitude and rhythm, which reflect the endurance capacity of dose and adaptive frequency of the individual, could be regarded as control basis of power and frequency [19]. In this system, the pulse wave signal is the basis of ultrasonic energy output and parameter adjustment.

The measured arterial pulse wave is formed by the blood spreading in the arterial circulation. The ascending branch of the wave reflects the passive expansion of the artery at the ventricular ejection period. The descending branch reflects the retraction of late-stage ejection. Furthermore, the dicrotic notch and dicrotic wave reflect the arterial elasticity and blood flow status. The variation regularity of blood volume and basic physiological status of a patient can be obtained through the identification and analysis of these features, allowing for amplitude-frequency selection and time division control based on physiological information [20].

In the process of pulse signal acquisition, there is inevitable baseline drift due to involuntary body movement and breathe, in addition to high-frequency noise and pseudo-peaks due to electromagnetic interference, as shown in Fig. 6a. In this study, the wavelet transform was applied to the signal from filtering and amplification and subsequently employed for analysis and modulation.

The ultrasonic control signal is modulated to the processed pulse wave as the carrier signal, which is shown in Eq. (10), where the modulated waveform is $F(t)$ and the pulse wave is $m(t)$ :

$$
F(t)=m(t) *(1+f(t)) \text {. }
$$

After a series of processes as described, the final ultrasonic control signal contains pulse wave information, as shown in Fig. 6b. Through modulation, the output amplitude is restricted to a moderate grade while the ultrasonic transducer is set at the optimal working status from frequency selection, thereby stabilizing the output.

\subsection{Output control based on blood volume}

In ultrasound hyperthermia, ultrasound energy is absorbed by the tissue and converted into heat. In healthy tissues, most of the heat produced is dispersed through blood circulation; however, tumors and inflamed tissue with typically inadequate blood supply retain a great deal of heat, which leads to the destruction of cell structure and growth inhibition, as shown in Fig. 7.

In this design, the dosage control is based on periodic changes in the volume of arterial blood. When the blood volume is high, the corresponding high ultrasonic irradiation is applied to destroy the tumor 


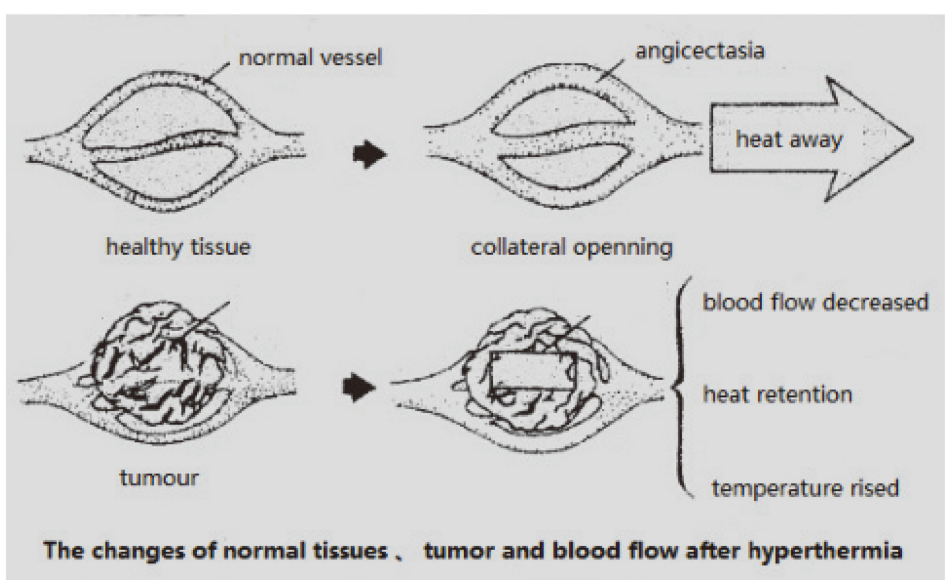

Fig. 7. Schematic diagram of tumor and healthy tissue after ultrasonic hyperthermia.

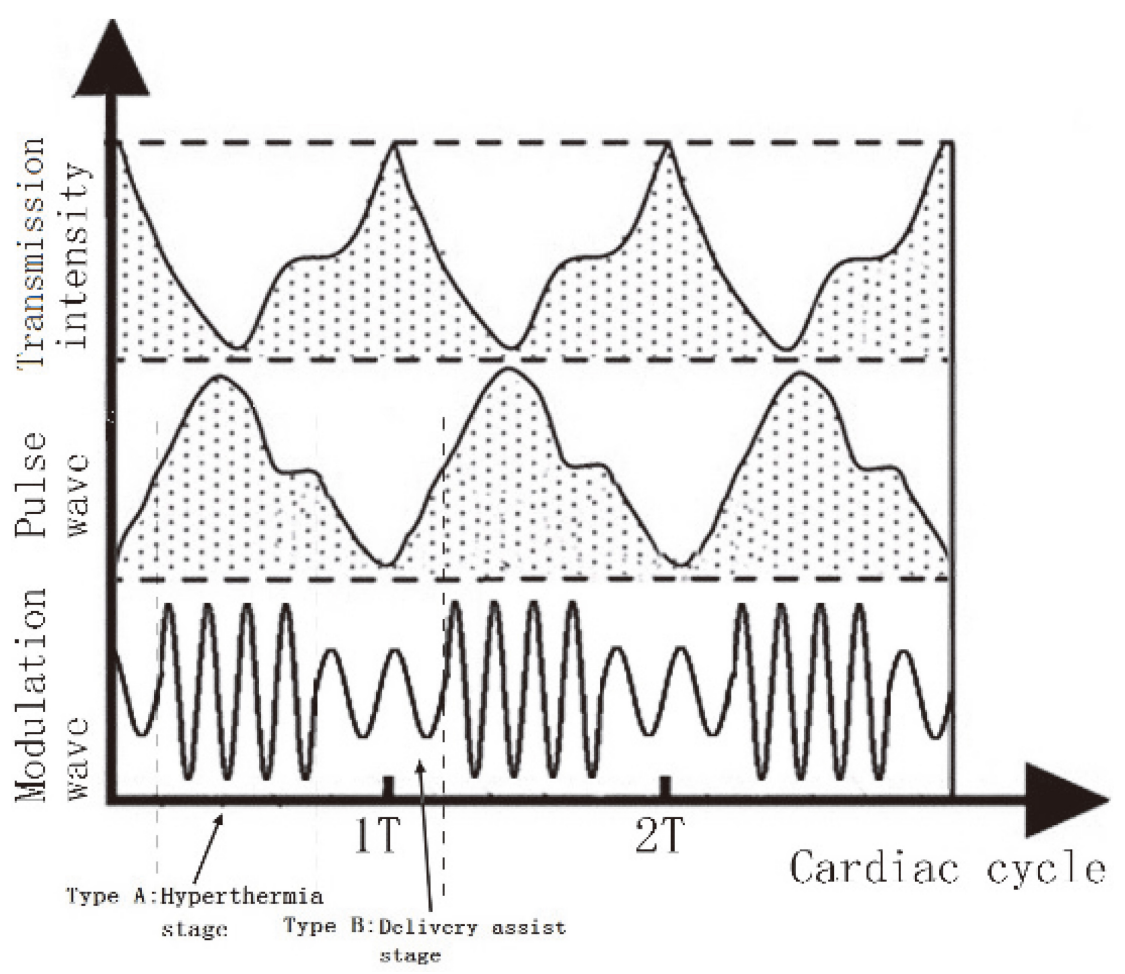

Fig. 8. Comparison between alternating frequency and pulse wave.

and inflammatory tissues, enabling the thermal radiation of normal tissues to be dispersed by the circulation of the blood. During the low-volume stage, the specified ultrasound frequency and pulse are set to assist particular drug penetration, as shown in Fig. 8.

By adjusting the time window and intensity of multi-frequency ultrasound, the irradiation dose was reduced to around $22 \%$ of that during continuous irradiation at $1 \mathrm{MHz}$, with an acoustic intensity of $2.0 \mathrm{~W} / \mathrm{cm}^{2}$, which still offers considerable treatment benefits. The alternate ratio of Type A: Type B is 
2:8, as shown in Fig. 8. For topical drug penetration, alternating frequency ultrasound therapy would be more effective, while assisting lesion rehabilitation through hyperthermia.

\section{Conclusions}

At this stage, the functional verifications of the system have been completed but clinical experiments have not yet been conducted. The results of this study show that the system is capable of pulse wave real-time acquisition and power control with the pulse modulated ultrasonic signal, which achieves synchronization of the acoustic effect and the human cardiovascular system.

In addition to the methods described above, the system output could be adjusted to deliver a short burst of high-power ultrasonic pulse appropriate to the treatment site and the individual characteristics of the patient. This mechanism would further actualize the dosage control and system optimization. The mechanism research would be phased in follow-up work.

This system accomplishes the synchronization of the ultrasonic output dose with physiological rhythms as well as the accurate and reliable control of treatment patterns and cycles, enabling smarter and more effective ultrasonic rehabilitation therapy.

\section{Acknowledgments}

The authors would like to thank the University Innovation Team Construction Plan Funding Project of Chongqing (Smart Medical System and Key Techniques). This work is partially supported by the National Science Foundation of China (Grant nos. 61601072, 61571070), Chongqing Key Laboratory Improvement Plan (Chongqing Key Laboratory of Photoelectronic Information Sensing and Transmitting Technology), Jiangsu Collaborative Innovation Center on Atmospheric Environment and Equipment Technology (CICAEET) Fund, China Postdoctoral Science Foundation (Grant nos. 171748) and Chongqing postdoctoral research project (Grant nos. Xm2016051).

\section{Conflict of interest}

None to report.

\section{References}

[1] Schoellhammer CM, Srinivasan S, Barman R, Mo SH, Polat B, Langer R, et al. Applicability and safety of dual-frequency ultrasonic treatment for the transdermal delivery of drugs. J Control Release. 2015; 202: 93-100.

[2] Sorace AG, Warram JM, Umphrey H, Hoyt K. Microbubble-mediated ultrasonic technique for improved chemotherapeutic delivery in cancer. J Drug Target. 2012; 20(1): 43-54.

[3] Okunaga S, Takasu A, Meshii N, Imai T, Hamada M, Iwai S, et al. Ultrasound as a method to enhance antitumor ability of oncolytic herpes simplex virus for head and neck cancer. Cancer Gene Ther. 2015; 22(3): 163-168.

[4] Udroiu I. Ultrasonic drug delivery in Oncology. J BUON. 2015; 20(2): 381-90.

[5] Yang FY, Lee PY. Efficiency of drug delivery enhanced by acoustic pressure during blood-brain barrier disruption induced by focused Ultrasound. Int J Nanomedicine. 2012; 7(8): 2573-2582.

[6] Chen LJ, Lu CT, Zhao YZ, Du LN, Jin YG. Ultrasonic microbubbles for glioma-targeted drug delivery. Acta Pharmaceutica Sinica. 2015; 50(1); 99-103. 
[7] Wu SY, Chen CC, Tung YS, Olumolade OO, Konofagou EE. Effects of the microbubble shell physicochemical properties on ultrasound-mediated drug delivery to the brain. J Control Release. 2015; 212: 30-40.

[8] Boyaci A, Tutoglu A, Boyaci N, Aridici R, Koca I. Comparison of the efficacy of ketoprofen phonophoresis, ultrasound, and short-wave diathermy in knee osteoarthritis. Rheumatol Int. 2013; 33(11): 2811-2818.

[9] Keikhosravi A, Zahedi E, Attar HM, Aghajani H. Experimental Investigation of the Roles of Blood Volume and Density in Finger Photoplethysmography. IEEE Sensors Journal. 2013; 13(5): 1397-1398.

[10] Zama MM, Ansari MM, Dimri U, Hoque M, Maiti SK, Kinjavderkar P. Effect of therapeutic ultrasound and diathermy on oxidant-antioxidant balance in dogs suffering from hind quarter weakness. Journal of Applied Animal Research. 2013; 41(1): 82-86.

[11] Szlosek PA, Taggart J, Cavallario JM, Hoch JM. Effectiveness of diathermy in comparison with ultrasound or corticosteroids in patients with tendinopathy: a critically appraised topic. J Sport Rehabil. 2014; 23(4): 370-375.

[12] Sutton JT, Haworth KJ, Pyne-Geithman G, Holland CK. Ultrasound-mediated drug delivery for cardiovascular disease. Expert Opin Drug Deliv. 2013; 10(5): 573-592.

[13] Goertz D, Hynynen K. Ultrasound-mediated drug delivery. Physics Today. 2016; 69(3): 30-6.

[14] Zhang C, Huang P, Zhang Y, Chen J, Shentu W, Sun Y, et al. Anti-tumor efficacy of ultrasonic cavitation is potentiated by concurrent delivery of anti-angiogenic drug in colon cancer. Cancer Lett. 2014; 347(1): 105-113.

[15] Xu YH, Zhou JZ, Xue XM, Fu WL, Zhu WL, Li CS. An adaptively fast fuzzy fractional order PID control for pumped storage hydro unit using improved gravitational search algorithm. Energy Conversion And Management. 2016; 111: 67-78.

[16] Liu K, Guo GK, Xiao YD, Tian SL. Analysis of Phase Truncation Error Based on Multi-Path Pseudo-Interleaved Direct Digital Frequency Synthesis. Journal of Circuits Systems And Computers. 2015; 24(10): 1550146.

[17] Alvarez J, Kumar N, Bettotti P, Hill D. Phase-Sensitive Detection for Optical Sensing With Porous Silicon. IEEE Photonics Journal. 2012; 4(3): 986-995.

[18] Sorace AG, Warram JM, Umphrey H, Hoyt K. Microbubble-mediated ultrasonic techniques for improved chemotherapeutic delivery in cancer. J Drug Target. 2012; 20(1): 43-54.

[19] Sahadan DZ, Davey MJ, Horne RS, Nixon GM. Improving detection of obstructive sleep apnoea by overnight oximetry in children using pulse rate parameters. Sleep Breath. 2015; 19(4): 1409-1414.

[20] Maria VC, Vera G, Giuseppe FS, Attilio R, Gionata F. Influence of Aortic Outflow Cannula Orientation on Epiaortic Flow Pattern During Pulsed Cardiopulmonary Bypass. Journal of Medical and Biological Engineering. 2015; 35(4): 455-463. 